\title{
Fate of Komadugu River Basin, Yobe State, Nigeria With Respect to Herbicides Residues
}

\author{
Mohammed $\mathrm{Al}^{*}$, Akan JC, Chellube $\mathrm{Z}$ and Inuwa LB
}

Department of Chemistry, University in Maiduguri, Nigeria

\begin{abstract}
Water, sediment and tissues of fish samples Clarias anguillaris, Tilapia zilli, Synodontis budgetti and Heterotis niloticus were collected during two seasons rainy and dry from Komadugu river basin, Yobe State, Nigeria for some herbicide residues determination. Extraction of herbicide residues and clean-up of fish samples were carried out using standard procedures. The concentration of the studied herbicide residues was determined using HPLC. The highest levels of all the studied herbicides were observed in the liver and the gills of the four species of fish analyzed, while the lowest value was observed in the flesh of all the studied species of fish. Accumulation of herbicides in the studied fish species were in the order of Synodontis budgetti>Heterotis niloticus >Tilapia zilli>Clarius anguillarias. Similarly, accumulation of herbicides in the studied tissues of the fish samples were in the order of liver>gills $>$ intestine $>$ flesh. All the studied herbicides were found to be within the ADI value of $0.05 \mathrm{mg} / \mathrm{kg}$ in fish and $0.01 \mathrm{mg} / \mathrm{kg} \mathrm{MRLs}$ in fish samples.
\end{abstract}

Keywords: Herbicides; Fish; Species; Water; Sediment; Komadugu

\section{Introduction}

Pesticides are defined as substances or mixtures of chemical substances meant primarily for killing, preventing, destroying, repelling, or mitigating any pest [1]. Pests can be insects, mice and other animals, unwanted plants (weeds), fungi, or microorganisms such as bacteria and viruses. Pesticides are employed to protect plants or other plant products from harmful organisms; to regulate the healthy growth of plants; to give protection against harmful creatures; or to render such creatures harmless [2]. Most pesticides may be harmful to the ecosystem because of their ecotoxicity, potential bio accumulating properties or their hormone disrupting effects. When pesticides are applied on the field, only a certain percentage of the applied dose will reach the target crop. Pesticides, especially herbicides are used quite extensively in most farming systems. The negative effect is that when herbicides are applied to the field they do not only control targeted weeds, but may also leave unwanted residues in the soil, which are ecologically harmful $[3,4]$. Although the efficacy of herbicides in controlling weeds is very high and applauded, its residual impact which might have health impact should also be considered for environmental safety. Preferred herbicides should not only have good efficacy for repelling pests, but also pose minimum adverse effects on crops, ecology and the ecosystem [5].

Paraquat, glyphosate and glufosinate-ammonium are among the most commonly applied herbicides [6]. Usage of these herbicides in the oil palm plantation causes damage on the environment (soil, water, and air) and adverse effects to non-targeted aquatic and terrestrial organisms [7]. Contamination of soil, water resources and agricultural products by herbicides is an increasing environmental concern [8]. Bioassay and chromatography are among the several methods commonly adopted in determining pesticide residue [7].

Globally, humans activities have altered terrestrial fluxes of freshwater [9] sediment [10] and nutrients [11,12] to coastal marine waters, including coral reef environments. Freshwater flow regimes have been modified through land use change which includes deforestation, wetland drainage, aquifer mining, surface water diversion and dam construction [9]. Sediment fluxes have increased as a result of soil erosion associated with deforestation, coastal development, agricultural practices and mining, whilst reductions are primarily due to retention within impoundments [9]. Nitrogen and phosphorus fluxes have increased due to changes in land use, agricultural crop and livestock production, discharge of urban and industrial wastewater and fossil fuel burning [11].

Consequent declines in reef water quality and other anthropogenic activities have resulted in detrimental impacts on physical, ecological and physiological processes of reef-building corals [13,14]. Extended exposure to lowered salinities associated with increased freshwater flows leads to reduced coral growth, coral bleaching, disease and mortality. Long term or high levels of sedimentation are harmful to all coral life history stages, including settlement of larvae, survival of recruits and growth of adults. Nutrient enrichment increases phytoplankton biomass, abundance of macro algae, risk of coral bleaching and prevalence of coral diseases. High phytoplankton biomass may facilitate population outbreaks of the coral-eating crown of thorns starfish $[15,16]$. Increased turbidity, caused by suspended sediment or phytoplankton blooms, reduces benthic light availability for photosynthesis and hence coral productivity. Overall, the effect of a long-term decline of reef water quality is loss of coral diversity, structure and function $[13,14]$. Reduction of land-based pollution has been widely advocated to reverse coral reef degradation [16] and enhance the resilience of the preferred, coral-reef dominated state $[17,18]$, particularly in the face of more extreme perturbation events forecast with climate change [19]. To ensure the future of coral reefs, the 2012 Consensus Statement on Climate Change and Coral Reefs has called for management of anthropogenic pressures including reducing landbased pollution [20]. To achieve such reductions in riverine pollutant

"Corresponding author: Mohammad Al, Department of Chemistry, University in Maiduguri, Nigeria, Tel: +2348036000506; E-mail: abdullahiim91@gmail.com

Received October 03, 2018; Accepted October 16, 2018; Published October 30 2018

Citation: Mohammed Al, Akan JC, Chellube Z, Inuwa LB (2018) Fate of Komadugu River Basin, Yobe State, Nigeria With Respect to Herbicides Residues. J Pet Environ Biotechnol 9: 383. doi: 10.4172/2157-7463.1000383

Copyright: (c) 2018 Mohammed Al, et al. This is an open-access article distributed under the terms of the Creative Commons Attribution License, which permits unrestricted use, distribution, and reproduction in any medium, provided the original author and source are credited. 
loads to coastal marine waters, it is critical to identify the main sources and pathways of pollutants.

The sources of water into the study area are from river Kano and river Jos. A lot of commercial agricultural activities take place along the banks of these rivers and in other to improve crop yield and eliminate weeds, majority of herbicides are used for weed control in order to boost and enhance productivity. Consequently, residues from the use of these herbicides are washed into the river which subsequently flows into the study area and fish from the river supplement the protein requirements of the inhabitants within the study area and other states. The water used by the populace for domestic and irrigation farming within the study area also comes from this river. Thus, the contamination of fish and the aquatic environment by herbicides is viewed with serious concern. Therefore, fish, sediment and water may accumulate herbicides in excessive amount in their various organs. This may ultimately, adversely negatively affect humans and other species that depend on such fish for food and water for domestic use. Recently, there has been growing concern over the water quality and safety of fish within the River Basin, hence, the need to evaluate the levels of some herbicides residues in the fish, sediment and water samples during two seasons dry and rainy.

\section{Materials and Methods}

\section{Fish sampling}

Four Fish samples were collected in accordance with the method described by Boyd et al. [21] namely Tilapia zilli, Clarias anguillaris, Synodontis budgetti and Heterotis niloticus were caught from Komadugu River Basin (Gashua and Nguru) through local fishermen. Fish samples of uniform size and weight were collected in order to avoid the possible error due to size differences. The fish samples were labeled with unique identification number and identified by an expert in the Department of Fisheries, University of Maiduguri and later dissected to remove tissues flesh, liver, intestine and gills of each species of fish and transferred into amber glass bottles and stored in an iced box pending further analysis.

\section{Extraction of fish samples for herbicide residues}

Extraction of fish sample was carried out in accordance with the method described by Paula et al. [22]. For the fish samples, 50g of the flesh, intestine, liver and gills of the fish from each sample were homogenized. Extractions were performed by using $150 \mathrm{ml}$ acetonitrile. The samples were filtered and rinsed twice with $25 \mathrm{ml}$ of the acetonitrile. The combined extract was concentrated using a rotary vacuum and evaporated over a hot water bath at $30^{\circ} \mathrm{C}$ to $50 \mathrm{ml}$. The liquid - liquid partitioning was transferred into a $500 \mathrm{ml}$ separating funnel and diluted with $250 \mathrm{ml}$ of $5 \%$ aqueous sodium chloride and partitioned into 150 and $100 \mathrm{ml}$ of $\mathrm{n}$-hexane. The combined $\mathrm{n}$-hexane layer was passed through anhydrous sodium sulphate and concentrated to near dryness of $10 \mathrm{ml} \mathrm{n}$-hexane. The levels of the studied herbicides were determined using GC-MS.

\section{Results and Discussion}

\section{Herbicide residues concentrations in water samples during rainy and dry seasons}

The concentrations of some herbicide residues in water samples from different sampling points of Komadugu river basin during the rainy and dry season are as presented in Tables 1 and 2. The concentration of Propachlor ranged from $1.00 \mathrm{E}-02$ to $2.50 \mathrm{E}-01 \mathrm{mg} / \mathrm{L} ; 1.50 \mathrm{E}-02$ to $4.60 \mathrm{E}-01 \mathrm{mg} / \mathrm{L}$ Atrazine; $1.00 \mathrm{E}-02$ to $1.60 \mathrm{E}-01 \mathrm{mg} / \mathrm{L}$ Propanil; $1.00 \mathrm{E}-02$

\begin{tabular}{|c|c|c|c|c|c|c|}
\hline \multicolumn{7}{|c|}{ Concentrations (mg/L) } \\
\hline $\begin{array}{l}\text { Sampling } \\
\text { Points }\end{array}$ & Propachlor & Atrazine & Propanil & Alachlor & Metolachlor & Butachlor \\
\hline S1 & $1.40 \mathrm{E}-01$ & 4.60E-01 & $1.20 \mathrm{E}-01$ & $1.30 \mathrm{E}-01$ & 7.00E-02 & $4.00 \mathrm{E}-02$ \\
\hline S2 & 8.00E-02 & $3.40 \mathrm{E}-01$ & $3.00 \mathrm{E}-02$ & $5.00 \mathrm{E}-02$ & 4.00E-02 & $2.00 \mathrm{E}-02$ \\
\hline S3 & $1.60 \mathrm{E}-01$ & $6.30 \mathrm{E}-01$ & $1.30 \mathrm{E}-01$ & $1.40 \mathrm{E}-01$ & 1.10E-01 & $6.00 \mathrm{E}-02$ \\
\hline S4 & $1.40 \mathrm{E}-01$ & $3.70 \mathrm{E}-01$ & $1.10 \mathrm{E}-01$ & 1.10E-01 & $6.00 \mathrm{E}-02$ & 4.00E-02 \\
\hline S5 & $1.80 \mathrm{E}-01$ & $4.60 \mathrm{E}-01$ & $1.40 \mathrm{E}-01$ & $1.50 \mathrm{E}-01$ & 8.00E-02 & 7.00E-02 \\
\hline S6 & 1.10E-01 & $1.60 \mathrm{E}-01$ & 8.00E-02 & 7.00E-02 & $5.00 \mathrm{E}-02$ & $6.00 \mathrm{E}-02$ \\
\hline S7 & $2.10 \mathrm{E}-01$ & $5.50 \mathrm{E}-01$ & $1.30 \mathrm{E}-01$ & $1.50 \mathrm{E}-01$ & $1.10 \mathrm{E}-01$ & 8.00E-02 \\
\hline S8 & $1.70 \mathrm{E}-01$ & 4.70E-01 & $1.40 \mathrm{E}-01$ & $1.50 \mathrm{E}-01$ & $9.00 \mathrm{E}-02$ & 1.10E-01 \\
\hline S9 & 1.10E-01 & $2.60 \mathrm{E}-01$ & 8.00E-02 & $9.00 \mathrm{E}-02$ & $5.00 \mathrm{E}-02$ & 5.00E-02 \\
\hline S10 & $2.50 \mathrm{E}-01$ & 4.30E-01 & $1.60 \mathrm{E}-01$ & $1.80 \mathrm{E}-01$ & 1.30E-01 & 8.00E-02 \\
\hline Total & $1.55 \mathrm{E}+00$ & $4.13 E+00$ & $1.12 \mathrm{E}+00$ & $1.22 \mathrm{E}+00$ & 7.90E-01 & $6.10 \mathrm{E}-01$ \\
\hline
\end{tabular}

Table 1: Herbicide residues concentration in water samples from ten different sampling points of Komadugu river basin during the rainy season.

\begin{tabular}{|c|c|c|c|c|c|c|}
\hline \multicolumn{7}{|c|}{ Concentrations (mg/kg) } \\
\hline Sampling points & Propachlor & Atrazine & Propanil & Alachlor & Metolachlor & Butachlor \\
\hline S1 & 4.00E-02 & $5.00 \mathrm{E}-02$ & $2.50 \mathrm{E}-02$ & 4.00E-02 & 4.00E-02 & 4.00E-02 \\
\hline S2 & $1.50 \mathrm{E}-02$ & $3.00 \mathrm{E}-02$ & $2.50 \mathrm{E}-02$ & 4.00E-02 & 3.00E-02 & 3.50E-02 \\
\hline S3 & 3.00E-02 & $2.00 \mathrm{E}-02$ & $1.50 \mathrm{E}-02$ & $1.00 \mathrm{E}-02$ & $2.50 \mathrm{E}-02$ & $1.50 \mathrm{E}-02$ \\
\hline S4 & 3.50E-02 & $2.00 \mathrm{E}-02$ & 2.00E-02 & $2.50 \mathrm{E}-02$ & $1.00 \mathrm{E}-02$ & $1.00 \mathrm{E}-02$ \\
\hline S5 & $2.50 \mathrm{E}-02$ & $1.50 \mathrm{E}-02$ & $6.00 \mathrm{E}-02$ & $1.50 \mathrm{E}-02$ & $1.50 \mathrm{E}-02$ & $2.00 \mathrm{E}-02$ \\
\hline S6 & 1.50E-02 & $1.50 \mathrm{E}-01$ & $2.50 \mathrm{E}-02$ & $3.00 \mathrm{E}-02$ & $2.50 \mathrm{E}-02$ & $2.00 \mathrm{E}-02$ \\
\hline S7 & 4.00E-02 & $2.00 \mathrm{E}-02$ & 1.00E-02 & $1.50 \mathrm{E}-02$ & $2.00 \mathrm{E}-02$ & $1.50 \mathrm{E}-02$ \\
\hline S8 & 1.00E-02 & $1.50 \mathrm{E}-02$ & $2.50 \mathrm{E}-02$ & $2.00 \mathrm{E}-02$ & 1.00E-02 & $1.50 \mathrm{E}-02$ \\
\hline S9 & $1.50 \mathrm{E}-02$ & $2.50 \mathrm{E}-02$ & $1.50 \mathrm{E}-02$ & $1.00 \mathrm{E}-02$ & $2.00 \mathrm{E}-02$ & $1.50 \mathrm{E}-02$ \\
\hline S10 & $1.50 \mathrm{E}-02$ & $2.00 \mathrm{E}-02$ & $1.50 \mathrm{E}-02$ & 2.00E-02 & $1.50 \mathrm{E}-02$ & $1.00 \mathrm{E}-02$ \\
\hline Total & 2.40E-01 & $3.65 \mathrm{E}-01$ & 2.35E-01 & 2.25E-01 & 2.10E-01 & $1.95 \mathrm{E}-01$ \\
\hline
\end{tabular}

Table 2: Herbicide residue concentration in water samples from ten different sampling points of Komadugu river basin during the dry season. 
to $1.80 \mathrm{E}-01 \mathrm{mg} / \mathrm{L}$ Alachlor; $1.00 \mathrm{E}-02$ to $1.30 \mathrm{E}-01 \mathrm{mg} / \mathrm{L}$ Metolachlor; $1.00 \mathrm{E}-02$ to $1.00 \mathrm{E}-01 \mathrm{mg} / \mathrm{L}$ Butachlor. Atrazine indicates the highest total concentrations with a value of $4.13+00 \mathrm{mg} / \mathrm{L}$ during the rainy season, while Butalachlor recorded the lowest total concentrations with a value of $1.95 \mathrm{E}-01 \mathrm{mg} / \mathrm{L}$ during the dry season.

\section{Herbicide residues concentrations in sediment samples during rainy and dry seasons}

The concentrations of some herbicide residues in sediment samples from different sampling points of Komadugu river basin during the rainy and dry season are as presented in Tables 3 and 4 . The concentration of propachlor ranged from $1.00 \mathrm{E}-01$ to $1.20 \mathrm{E}+01 \mathrm{mg} / \mathrm{kg} ; 2.00 \mathrm{E}-02$ to $6.00 \mathrm{E}+01 \mathrm{mg} / \mathrm{kg}$ atrazine; $1.00 \mathrm{E}-01$ to $4.00 \mathrm{E}+01 \mathrm{mg} / \mathrm{kg}$ propanil; $8.00 \mathrm{E}-02$ to $1.00 \mathrm{E}+01 \mathrm{mg} / \mathrm{kg}$ alachlor; $8.00 \mathrm{E}-02$ to $2.23 \mathrm{E}+00 \mathrm{mg} / \mathrm{kg}$ metolachlor and $4.00 \mathrm{E}-02$ to $6.00 \mathrm{E}+00 \mathrm{mg} / \mathrm{kg}$ butachlor. propachlor shows the highest total concentration with a value of $8.05+01 \mathrm{mg} / \mathrm{L}$ during the rainy season, while Propanil recorded the lowest total concentrations with a value of $3.54 \mathrm{mg} / \mathrm{kg}$ during the dry season.

\section{Herbicide residues concentrations in different tissues of fish samples during rainy and dry seasons}

The concentrations of some herbicide residues in different tissues of Heterotis niloticus studied from Komadugu River Basin, Yobe State Nigeria during the rainy and dry seasons are as presented in Tables 5 and 6. The concentration of propachlor ranged from 1.00 E-04 to 9.00E$02 \mathrm{mg} / \mathrm{kg} ; 2.00 \mathrm{E}-03$ to $1.40 \mathrm{E}-01 \mathrm{mg} / \mathrm{kg}$ atrazine; $2.00 \mathrm{E}-04$ to $7.00 \mathrm{E}-02$ $\mathrm{mg} / \mathrm{kg}$ propanil; $1.00 \mathrm{E}-04$ to $9.00 \mathrm{E}-02 \mathrm{mg} / \mathrm{kg}$ alachlor; $1.00 \mathrm{E}-04$ to
$5.00 \mathrm{E}-02 \mathrm{mg} / \mathrm{kg}$ metolachlor; $1.50 \mathrm{E}-04$ to $4.50 \mathrm{E}-02 \mathrm{mg} / \mathrm{kg}$ Butachlor. Atrazine shows the highest total concentrations with a value of $3.80 \mathrm{E}$ $01 \mathrm{mg} / \mathrm{kg}$ during the rainy season, while metolachlor recorded the lowest total concentrations with a value of $1.00 \mathrm{E}-03 \mathrm{mg} / \mathrm{kg}$ during the dry season. For Synodontis budgetti, the concentration of propachlor ranged from $1.50 \mathrm{E}-03$ to $1.30 \mathrm{E}-01 \mathrm{mg} / \mathrm{kg} ; 1.50 \mathrm{E}-02$ to $1.80 \mathrm{E}-01 \mathrm{mg} / \mathrm{kg}$ atrazine; $1.50 \mathrm{E}-04$ to $8.00 \mathrm{E}-02 \mathrm{mg} / \mathrm{kg}$ propanil; $1.00 \mathrm{E}-04$ to $1.10 \mathrm{E}-01$ $\mathrm{mg} / \mathrm{kg}$ Alachlor; $1.50 \mathrm{E}-04$ to $7.00 \mathrm{E}-02 \mathrm{mg} / \mathrm{kg}$ Metolachlor; $1.50 \mathrm{E}-04$ to $6.00 \mathrm{E}-02 \mathrm{mg} / \mathrm{kg}$ Butachlor (Tables 7 and 8). Atrazine shows the highest total concentrations with a value of $5.20 \mathrm{E}-01 \mathrm{mg} / \mathrm{kg}$ during the rainy season, while propanil recorded the lowest total concentrations with a value of $6.85 \mathrm{E}-03 \mathrm{mg} / \mathrm{kg}$ during the dry season.

The concentrations of some herbicide residues in different tissues of Tilapia zilli studied from Komadugu River Basin, Yobe State Nigeria during the rainy and dry seasons are as presented in Tables 9 and 10. The concentration of Propachlor ranged from 1.50E-03 to 7.00E-02 $\mathrm{mg} / \mathrm{kg} ; 1.50 \mathrm{E}-03$ to $9.00 \mathrm{E}-02 \mathrm{mg} / \mathrm{kg}$ Atrazine; $2.50 \mathrm{E}-04$ to $9.00 \mathrm{E}-03 \mathrm{mg} /$ $\mathrm{kg}$ Propanil; $1.00 \mathrm{E}-03$ to $5.00 \mathrm{E}-03 \mathrm{mg} / \mathrm{kg}$ Alachlor; $2.00 \mathrm{E}-04$ to $8.00 \mathrm{E}-$ $03 \mathrm{mg} / \mathrm{kg}$ Metolachlor; $1.00 \mathrm{E}-04$ to $4.00 \mathrm{E}-03 \mathrm{mg} / \mathrm{kg}$ Butachlor. Atrazine shows the highest total concentrations with a value of $2.40 \mathrm{E}-01 \mathrm{mg} /$ $\mathrm{kg}$ during the rainy season, while butachlor recorded the lowest total concentrations with a value of $1.30 \mathrm{E}-03 \mathrm{mg} / \mathrm{kg}$ during the dry season. For Clarias anguilaris the concentration of propachlor ranged from $3.00 \mathrm{E}-04$ to $1.00 \mathrm{E}-01 \mathrm{mg} / \mathrm{kg} ; 5.00 \mathrm{E}-04$ to $6.00 \mathrm{E}-02 \mathrm{mg} / \mathrm{kg}$ Atrazine; $3.00 \mathrm{E}-04$ to $6.00 \mathrm{E}-03 \mathrm{mg} / \mathrm{kg}$ propanil; $1.00 \mathrm{E}-04$ to $4.00 \mathrm{E}-03 \mathrm{mg} / \mathrm{kg}$ alachlor; $1.00 \mathrm{E}-04$ to $5.00 \mathrm{E}-03 \mathrm{mg} / \mathrm{kg}$ metolachlor; $1.50 \mathrm{E}-04$ to $2.00 \mathrm{E}-$ $03 \mathrm{mg} / \mathrm{kg}$ butachlor (Tables 11 and 12). Atrazine shows the highest total

\begin{tabular}{|c|c|c|c|c|c|c|}
\hline \multicolumn{7}{|c|}{ Concentrations (mg/kg) } \\
\hline Sampling Points & Propachlor & Atrazine & Propanil & Alachlor & Metolachlor & Butachlor \\
\hline $\mathrm{S} 1$ & 2.30E-01 & $6.30 \mathrm{E}-01$ & $1.60 \mathrm{E}-01$ & $1.80 \mathrm{E}+01$ & $1.40 \mathrm{E}-01$ & 7.00E-02 \\
\hline $\mathrm{S} 2$ & 1.10E-01 & $2.00 \mathrm{E}-02$ & $1.00 \mathrm{E}-01$ & 8.00E-02 & 8.00E-02 & 4.00E-02 \\
\hline S3 & 2.30E-01 & $1.60 \mathrm{E}+01$ & $1.60 \mathrm{E}-01$ & $1.80 \mathrm{E}-01$ & $1.20 \mathrm{E}-01$ & 1.10E-01 \\
\hline S4 & $1.70 \mathrm{E}-01$ & $6.00 \mathrm{E}-01$ & $1.30 \mathrm{E}-01$ & $1.50 \mathrm{E}-01$ & $9.00 \mathrm{E}-02$ & $6.00 \mathrm{E}-02$ \\
\hline S5 & $2.40 \mathrm{E}-01$ & $6.11 \mathrm{E}-01$ & $1.70 \mathrm{E}-01$ & $2.10 \mathrm{E}-01$ & $1.30 \mathrm{E}-01$ & $1.10 \mathrm{E}-01$ \\
\hline S6 & $1.80 \mathrm{E}-01$ & $3.00 \mathrm{E}-01$ & $1.30 \mathrm{E}-01$ & $1.50 \mathrm{E}-01$ & $1.10 \mathrm{E}-01$ & $9.00 \mathrm{E}-02$ \\
\hline S7 & 3.20E-01 & $7.20 \mathrm{E}-01$ & $1.90 \mathrm{E}-01$ & $2.20 \mathrm{E}-01$ & $1.30 \mathrm{E}-01$ & 1.10E-01 \\
\hline S8 & $3.40 \mathrm{E}-01$ & 8.30E-01 & $2.10 \mathrm{E}-01$ & $2.70 \mathrm{E}-01$ & $1.60 \mathrm{E}-01$ & $1.30 \mathrm{E}-01$ \\
\hline S9 & $1.80 \mathrm{E}-01$ & $5.00 \mathrm{E}-01$ & $1.30 \mathrm{E}-01$ & $1.60 \mathrm{E}-01$ & $1.00 \mathrm{E}-01$ & 8.00E-02 \\
\hline $\mathrm{S} 10$ & 4.30E-01 & 7.02E-01 & $2.10 \mathrm{E}-01$ & $2.60 \mathrm{E}-01$ & $1.50 \mathrm{E}-01$ & $1.20 \mathrm{E}-01$ \\
\hline Total & $2.43 E+00$ & $2.09 \mathrm{E}+01$ & $1.59 \mathrm{E}+00$ & $1.97 \mathrm{E}+01$ & $1.21 \mathrm{E}+00$ & 9.20E-01 \\
\hline
\end{tabular}

Table 3: Herbicide residues concentration in sediment samples from ten different sampling points of Komadugu river basin during the rainy season.

\begin{tabular}{|c|c|c|c|c|c|c|}
\hline \multicolumn{7}{|c|}{ Concentrations (mg/kg) } \\
\hline Sampling Points & Propachlor & Atrazine & Propanil & Alachlor & Metolachlor & Butachlor \\
\hline S1 & $2.44 \mathrm{E}+00$ & $2.20 \mathrm{E}-01$ & $1.20 \mathrm{E}-01$ & $2.33 E+00$ & $1.00 \mathrm{E}-01$ & $1.07 E+00$ \\
\hline $\mathrm{S} 2$ & $3.31 \mathrm{E}+00$ & $5.40 \mathrm{E}-01$ & $1.00 \mathrm{E}+00$ & $4.11 \mathrm{E}+00$ & 2.20E-01 & $2.11 \mathrm{E}+00$ \\
\hline S3 & $7.22 \mathrm{E}+00$ & $1.22 \mathrm{E}+00$ & $2.44 \mathrm{E}+00$ & $8.23 E+00$ & $1.42 \mathrm{E}+00$ & $3.22 \mathrm{E}+00$ \\
\hline S4 & $5.00 \mathrm{E}+00$ & $2.00 \mathrm{E}+00$ & $1.00 \mathrm{E}+00$ & $4.00 \mathrm{E}+00$ & $7.60 \mathrm{E}-01$ & $3.00 \mathrm{E}+00$ \\
\hline S5 & $7.00 \mathrm{E}+00$ & $4.00 \mathrm{E}+00$ & $3.00 \mathrm{E}+00$ & $7.00 \mathrm{E}+00$ & $1.00 \mathrm{E}+00$ & $4.55 \mathrm{E}+00$ \\
\hline S6 & $1.20 \mathrm{E}+01$ & $6.00 \mathrm{E}+00$ & $4.00 \mathrm{E}+00$ & $1.00 \mathrm{E}+01$ & $2.23 \mathrm{E}+00$ & $6.00 \mathrm{E}+00$ \\
\hline S7 & $3.00 \mathrm{E}+00$ & $1.00 \mathrm{E}+00$ & $2.20 \mathrm{E}-01$ & $2.00 \mathrm{E}+00$ & $3.40 \mathrm{E}-01$ & $2.00 \mathrm{E}+00$ \\
\hline S8 & $5.00 \mathrm{E}+00$ & $2.00 \mathrm{E}+00$ & $1.00 \mathrm{E}+00$ & $4.00 E+00$ & $7.60 \mathrm{E}-01$ & $3.00 \mathrm{E}+00$ \\
\hline s9 & $7.00 \mathrm{E}+00$ & $4.00 \mathrm{E}+00$ & $3.00 \mathrm{E}+00$ & $7.00 \mathrm{E}+00$ & $1.00 \mathrm{E}+00$ & $4.55 E+00$ \\
\hline $\mathrm{S} 10$ & $1.22 \mathrm{E}+00$ & $1.10 \mathrm{E}-01$ & $6.00 \mathrm{E}-02$ & $1.18 \mathrm{E}+00$ & $5.00 \mathrm{E}-02$ & $6.00 \mathrm{E}-02$ \\
\hline Total & $5.32 \mathrm{E}+01$ & $2.11 \mathrm{E}+01$ & $1.58 \mathrm{E}+01$ & $4.99 E+01$ & $7.88 \mathrm{E}+00$ & $2.96 \mathrm{E}+01$ \\
\hline
\end{tabular}

Table 4: Herbicide residues concentration in sediment samples from ten different sampling points of Komadugu river basin during the dry season. 
Citation: Mohammed Al, Akan JC, Chellube Z, Inuwa LB (2018) Fate of Komadugu River Basin, Yobe State, Nigeria With Respect to Herbicides Residues. J Pet Environ Biotechnol 9: 383. doi: 10.4172/2157-7463.1000383

Page 4 of 7

\begin{tabular}{|c|c|c|c|c|c|}
\hline \multicolumn{2}{|c|}{} & \multicolumn{3}{|c|}{ Concentrations (mg/kg) } \\
\hline Tissues & Propachlor & Atrazine & Propanil & Alachlor & Metolachlor \\
\hline Flesh & $2.00 \mathrm{E}-02$ & $5.00 \mathrm{E}-02$ & $1.00 \mathrm{E}-02$ & $4.00 \mathrm{E}-02$ & $1.00 \mathrm{E}-02$ \\
\hline Gill & $9.00 \mathrm{E}-02$ & $1.20 \mathrm{E}-01$ & $5.00 \mathrm{E}-02$ & $7.00 \mathrm{E}-02$ & $3.00 \mathrm{E}-02$ \\
\hline Intestine & $3.00 \mathrm{E}-02$ & $7.00 \mathrm{E}-02$ & $2.00 \mathrm{E}-02$ & $4.00 \mathrm{E}-02$ & $1.00 \mathrm{E}-02$ \\
\hline Liver & $8.00 \mathrm{E}-02$ & $1.40 \mathrm{E}-01$ & $6.00 \mathrm{E}-02$ & $9.00 \mathrm{E}-02$ & $5.00 \mathrm{E}-02$ \\
\hline Total & $2.20 \mathrm{E}-01$ & $3.80 \mathrm{E}-01$ & $1.40 \mathrm{E}-01$ & $2.40 \mathrm{E}-01$ & $1.00 \mathrm{E}-01$ \\
\hline
\end{tabular}

Table 5: Herbicide residues concentration in different tissues of fish samples of Heterotis niloticus from Komadugu river basin during the rainy season.

\begin{tabular}{|c|c|c|c|c|c|c|}
\hline \multicolumn{7}{|c|}{ Concentrations (mg/kg) } \\
\hline Tissues & Propachlor & Atrazine & Propanil & Alachlor & Metolachlor & Butachlor \\
\hline Flesh & 1.00E-03 & $2.00 \mathrm{E}-03$ & 1.00E-03 & 1.00E-04 & $1.00 \mathrm{E}-03$ & $1.50 \mathrm{E}-04$ \\
\hline Gill & 1.00E-02 & $9.00 \mathrm{E}-02$ & 7.00E-02 & $5.00 \mathrm{E}-03$ & 5.00E-03 & $7.50 \mathrm{E}-03$ \\
\hline Intestine & $1.00 \mathrm{E}-04$ & 3.00E-03 & $2.00 \mathrm{E}-04$ & 2.00E-04 & $1.00 \mathrm{E}-04$ & $2.50 \mathrm{E}-03$ \\
\hline Liver & $6.00 \mathrm{E}-03$ & $5.00 \mathrm{E}-02$ & $4.00 \mathrm{E}-04$ & $3.00 \mathrm{E}-04$ & $2.00 \mathrm{E}-03$ & 4.50E-02 \\
\hline Total & $1.71 \mathrm{E}-02$ & $1.45 \mathrm{E}-01$ & $1.40 \mathrm{E}-03$ & $5.60 \mathrm{E}-03$ & $1.00 \mathrm{E}-03$ & $5.52 \mathrm{E}-02$ \\
\hline
\end{tabular}

Table 6: Herbicide residues mean concentrations in different tissues of fish samples of Heterotis niloticus from Komadugu river basin during the dry season.

\begin{tabular}{|c|c|c|c|c|c|c|}
\hline \multicolumn{7}{|c|}{ Concentrations (mg/kg) } \\
\hline Tissues & Propachlor & Atrazine & Propanil & Alachlor & Metolachlor & Butachlor \\
\hline Flesh & 4.00E-02 & 8.00E-02 & $2.00 \mathrm{E}-02$ & 5.00E-02 & 2.00E-02 & 3.00E-02 \\
\hline Gill & $1.10 \mathrm{E}-01$ & $1.50 \mathrm{E}-01$ & 7.00E-02 & $9.00 \mathrm{E}-02$ & $5.00 \mathrm{E}-02$ & 4.00E-02 \\
\hline Intestine & $6.00 \mathrm{E}-02$ & 1.10E-01 & $4.00 \mathrm{E}-02$ & 6.00E-02 & 3.00E-02 & $2.00 \mathrm{E}-02$ \\
\hline Liver & $1.30 \mathrm{E}-01$ & $1.80 \mathrm{E}-01$ & 8.00E-02 & 1.10E-01 & 7.00E-02 & $6.00 \mathrm{E}-02$ \\
\hline Total & $3.40 \mathrm{E}-01$ & $5.20 \mathrm{E}-01$ & 2.10E-01 & 3.10E-01 & $1.70 \mathrm{E}-01$ & $1.50 \mathrm{E}-01$ \\
\hline
\end{tabular}

Table 7: Herbicide residues mean concentrations in different tissues of fish samples of Synodontis budgetti from Komadugu river basin during the rainy season.

\begin{tabular}{|c|c|c|c|c|c|c|}
\hline \multicolumn{7}{|c|}{ Concentrations (mg/kg) } \\
\hline Tissues & Propachlor & Atrazine & Propanil & Alachlor & Metolachlor & Butachlor \\
\hline Flesh & $1.50 \mathrm{E}-03$ & $1.50 \mathrm{E}-02$ & $1.50 \mathrm{E}-04$ & $1.00 \mathrm{E}-04$ & $1.50 \mathrm{E}-04$ & $1.50 \mathrm{E}-04$ \\
\hline Gill & $6.50 \mathrm{E}-02$ & $9.00 \mathrm{E}-02$ & $\begin{array}{l}6.00 \mathrm{E}-03 \\
6.00 \mathrm{E}-03\end{array}$ & $5.50 \mathrm{E}-03$ & $7.50 \mathrm{E}-03$ & $6.00 \mathrm{E}-03$ \\
\hline Intestine & $2.50 \mathrm{E}-03$ & $2.50 \mathrm{E}-02$ & $2.00 \mathrm{E}-04$ & $2.00 \mathrm{E}-03$ & $2.50 \mathrm{E}-04$ & 2.00E-04 \\
\hline Liver & $3.00 \mathrm{E}-02$ & 4.00E-02 & $5.00 \mathrm{E}-04$ & $3.00 \mathrm{E}-03$ & $5.00 \mathrm{E}-03$ & $4.00 \mathrm{E}-03$ \\
\hline Total & $9.90 \mathrm{E}-02$ & $1.70 \mathrm{E}-01$ & $6.85 \mathrm{E}-03$ & $1.06 \mathrm{E}-02$ & 1.29E-02 & 1.04E-02 \\
\hline
\end{tabular}

Table 8: Herbicide residues mean concentrations in different tissues of fish samples of Synodontis budgetti from Komadugu river basin during the dry season.

\begin{tabular}{|c|c|c|c|c|c|c|}
\hline \multicolumn{7}{|c|}{ Concentraions (mg/kg) } \\
\hline Tissues & Propachlor & Atrazine & Propanil & Alachlor & Metolachlor & Butachlor \\
\hline Flesh & $2.00 \mathrm{E}-02$ & $3.00 \mathrm{E}-02$ & 3.00E-03 & $1.00 \mathrm{E}-03$ & $3.00 \mathrm{E}-03$ & 1.00E-03 \\
\hline Gill & 6.00E-02 & 8.00E-02 & $9.00 \mathrm{E}-03$ & $5.00 \mathrm{E}-03$ & $6.00 \mathrm{E}-03$ & 3.00E-03 \\
\hline Intestine & 3.00E-02 & 4.00E-02 & 4.00E-03 & $2.00 \mathrm{E}-03$ & 2.00E-03 & 1.00E-03 \\
\hline Liver & 7.00E-02 & $9.00 \mathrm{E}-02$ & 7.00E-03 & 4.00E-03 & 8.00E-03 & 4.00E-03 \\
\hline Total & $1.80 \mathrm{E}-01$ & $2.40 \mathrm{E}-01$ & $2.30 \mathrm{E}-02$ & $1.20 \mathrm{E}-02$ & $1.90 \mathrm{E}-02$ & $9.00 \mathrm{E}-03$ \\
\hline
\end{tabular}

Table 9: Herbicide residues mean concentrations in different tissues of fish samples of Tilapia zilli from Komadugu river basin, during the rainy season.

\begin{tabular}{|c|c|c|c|c|c|c|}
\hline \multicolumn{7}{|c|}{ Concentraions (mg/kg) } \\
\hline Tissues & Propachlor & Atrazine & Propanil & Alachlor & Metolachlor & Butachlor \\
\hline Flesh & $1.50 \mathrm{E}-03$ & $1.50 \mathrm{E}-03$ & $1.50 \mathrm{E}-03$ & $1.00 \mathrm{E}-03$ & $1.00 \mathrm{E}-03$ \\
\hline Gill & $6.50 \mathrm{E}-03$ & $8.00 \mathrm{E}-03$ & $7.50 \mathrm{E}-03$ & $4.50 \mathrm{E}-03$ & $7.50 \mathrm{E}-03$ \\
\hline Intestine & $2.50 \mathrm{E}-03$ & $2.50 \mathrm{E}-03$ & $2.50 \mathrm{E}-04$ & $1.50 \mathrm{E}-03$ & $2.00 \mathrm{E}-04$ \\
\hline Liver & $4.00 \mathrm{E}-04$ & $4.00 \mathrm{E}-02$ & $3.50 \mathrm{E}-03$ & $2.50 \mathrm{E}-03$ & $4.00 \mathrm{E}-04$ \\
\hline Total & $1.09 \mathrm{E}-02$ & $5.20 \mathrm{E}-02$ & $1.50 \mathrm{E}-04$ & 9.04 & $9.50 \mathrm{E}-03$ & $9.10 \mathrm{E}-03$ \\
\hline
\end{tabular}

Table 10: Herbicide residues mean concentrations in different tissues of fish samples of Tilapia zilli from Komadugu river basin, during the dry season 


\begin{tabular}{|c|c|c|c|c|c|c|}
\hline \multicolumn{7}{|c|}{ Concentrations (mg/kg) } \\
\hline Tissues & Propachlor & Atrazine & Propanil & Alachlor & Metolachlor & Butachlor \\
\hline Flesh & $1.00 \mathrm{E}-01$ & $3.00 \mathrm{E}-02$ & $1.00 \mathrm{E}-03$ & $1.00 \mathrm{E}-03$ & $2.00 \mathrm{E}-03$ \\
\hline Gill & $4.00 \mathrm{E}-02$ & $6.00 \mathrm{E}-02$ & $6.00 \mathrm{E}-03$ & $4.00 \mathrm{E}-03$ & $3.00 \mathrm{E}-03$ \\
\hline Intestine & $2.00 \mathrm{E}-03$ & $3.00 \mathrm{E}-02$ & $2.00 \mathrm{E}-03$ & $1.00 \mathrm{E}-03$ & $1.00 \mathrm{E}-03$ \\
\hline Liver & $3.00 \mathrm{E}-02$ & $4.00 \mathrm{E}-02$ & $4.00 \mathrm{E}-03$ & $3.00 \mathrm{E}-03$ & $5.00 \mathrm{E}-03$ \\
\hline Total & $1.72 \mathrm{E}-01$ & $1.60 \mathrm{E}-01$ & $1.00 \mathrm{E}-03$ & $9.00 \mathrm{E}-03$ & \\
\hline
\end{tabular}

Table 11: Herbicide residues mean concentrations in different tissues of fish samples of clarius anguillari from Komadugu river basin, during the rainy season

\begin{tabular}{|c|c|c|c|c|c|c|}
\hline \multicolumn{7}{|c|}{ Concentrations (mg/kg) } \\
\hline Tissues & Propachlor & Atrazine & Propanil & Alachlor & Metolachlor & $1.00 \mathrm{E}-04$ \\
\hline Flesh & $3.00 \mathrm{E}-04$ & $2.00 \mathrm{E}-03$ & $3.00 \mathrm{E}-04$ & $1.00 \mathrm{E}-04$ & $1.50 \mathrm{E}-04$ \\
\hline Gill & $4.50 \mathrm{E}-04$ & $7.50 \mathrm{E}-04$ & $6.20 \mathrm{E}-03$ & $1.00 \mathrm{E}-03$ & $2.00 \mathrm{E}-03$ \\
\hline Intestine & $4.00 \mathrm{E}-04$ & $5.00 \mathrm{E}-04$ & $5.00 \mathrm{E}-04$ & $2.00 \mathrm{E}-04$ & $2.00 \mathrm{E}-04$ \\
\hline Liver & $5.00 \mathrm{E}-04$ & $8.30 \mathrm{E}-03$ & $7.40 \mathrm{E}-03$ & $5.00 \mathrm{E}-04$ & $3.00 \mathrm{E}-03$ \\
\hline Total & $1.65 \mathrm{E}-03$ & $1.16 \mathrm{E}-02$ & $1.44 \mathrm{E}-02$ & $1.80 \mathrm{E}-03$ & 2.03 \\
\hline
\end{tabular}

Table 12: Herbicide residues mean concentrations in different tissues of fish samples of Clarius anguillaris from Komadugu river basin during the dry season.

concentrations with a value of $1.60 \mathrm{E}-01 \mathrm{mg} / \mathrm{kg}$ during the rainy season, while alachlor recorded the lowest total concentrations with a value of $1.80 \mathrm{E}-03 \mathrm{mg} / \mathrm{kg}$ during the dry season.

\section{Herbicide residues in water and sediment samples}

The levels of all the studied herbicides were significantly higher at point S7, while point S4 indicates the lowest concentrations. All the studied herbicides in the water samples were dominant during the rainy season with the highest concentration of $5.50 \mathrm{E}-01 \mathrm{mg} / \mathrm{l}$, while the dry season showed the lowest value of $1.00 \mathrm{E}-02 \mathrm{mg} / \mathrm{l}$. For sediment samples, the highest concentrations of all the herbicides were detected at point S3, while the lowest concentrations fluctuated between the ten sampling points. Atrazine was also the most dominant herbicide detected in the sediment sample with a value of $1.60 \mathrm{E}+01 \mathrm{mg} / \mathrm{kg}$. The concentrations of all the studied herbicides were higher in the sediment samples when compared with water samples as mentioned earlier. This is because of the hydrophobic characteristic of pesticides. It is expected that any herbicide present in the study area preferably bound to the particle phase in the aquatic system and then accumulated to the sediment through sedimentation process. Herbicides have an affinity for particulate matter and one of their main sinks is thought to be river and lake sediments [23].

Herbicides is adsorbed onto suspended matter in water, and onto sediment, with "no evidence of desorption back into the water phase" [24]. According to Eisler [24], loss of herbicides from the water phase is rapid: about $50 \%$ in $36 \mathrm{hr}$ and $100 \%$ in 4 weeks from freshwater ecosystems; and in marine ecosystems, $50-70 \%$ loss of herbicides from seawater was usually recorded within $24 \mathrm{hr}$. It moves from the water itself onto aquatic weeds, sediment and suspended solids. In a study of surface waters in a wetland in Spain, paraquat was found in $6.6 \%$ of samples from a lagoon (maximum level $3.95 \mu \mathrm{g} / \mathrm{L}$ ), and in $9.35 \%$ of samples from a marsh (maximum level $1.45 \mu \mathrm{g} / \mathrm{L}$ ) [25].

The levels of atrazine, propanil, alachlor and metolachlor in the water and sediment samples fluctuate within all the sampling points. Relatively, the concentrations of atrazine, propanil, alachlor and metolachlor were significantly higher in the sediment samples, while the water sample shows the lowest concentrations. This trend is due to the hydrophobic characteristic of pesticides.

The concentrations of atrazine, propanil, alachlor, metolachlor and butachlor in the water samples from the ten sampling points were lower than the set EU maximum residue limit (MRL) of $0.1 \mathrm{mg} / \mathrm{l}$ during the dry season, while results for the rainy season were above the MRL limit. Similarly, the concentrations of atrazine, propanil and metolachlor detected in the sediment samples were lower than the set EU maximum residue limit (MRL) of $5 \mathrm{mg} / \mathrm{kg}$ [1], with exception of propachlor and alachlor during the dry season which were higher than the set limit. Hence, the result indicates that the water and sediment samples within the study area were not contaminated by atrazine, propanil, metolachlor and butachlor.

\section{Herbicide residues in fish samples}

Herbicides tend to accumulate in living organisms especially in aquatic organisms and they substantially settle on the sediments [26]. The highest concentrations of all the studied herbicides were observed in the liver and the gills of the four species of fish analyzed, while the lowest value was observed in the flesh of all the studied species of fish. Despite the adverse effects of pesticides, herbicides form an integral component of modern agricultural practice. The benefits of herbicides are increased supply of food, but problems arise when a significant amount of the chemicals are left on the field as residue which tend to affect non target organisms and river bodies which are the main recipient of pesticide residues generated on the field. The concentrations of atrazine were significantly higher in the gills of the three species of fish studied. Atrazine is one of the most widely used herbicides globally, commonly used in Nigeria for the control of weeds in most farms [27]. Despite its intensive use, atrazine has been implicated in a number of health effects [28]. High concentrations of atrazine in serum and urine samples of cattle were observed by Peighambarzadeh et al. [29], with concentrations ranging from 0.739 and $1.389 \mu \mathrm{g} / \mathrm{l}$ respectively. In the present study the highest concentration of atrazine was observed in the gills and liver, while the flesh showed the lowest values. The high levels of atrazine in the gills might be attributed to the fact that the gills serve as the main route for the entrance of pollutants into the fish body. The concentration of butachlor and alachlor were higher in the gills, while the flesh showed the lowest value. A study carried out by He et al. [30], showed that butachlor poses a potential threat to the aquatic ecosystem. When the individual and joint toxicities of three chloroacetanilide herbicides, such as alachlor, acetochlor and butachlor, to a fresh water cladoceran Daphnia carinata was assessed, the common specific target site of these herbicides, which inhibit the synthesis of very long-chain fatty acids was revealed. Butachlor has been proved to be genotoxic and cytotoxic in catfish, Clarias batrachus, and the 
concentration of butachlor was found to be proportional to the extent of DNA damage [31]. In another study, butachlor was found to cause remarkable protein loss in C. batrachus at both lethal and sub-lethal concentrations. This might be due to increased proteolysis [32] or by metabolic utilization of ketoacids to glucogenesis pathway for synthesis of glucose [33]. The results demonstrated adverse effects of butachlor on the normal reproductive process of zebra fish and it was also found to disrupt the thyroid and sex steroid (endocrine) systems when exposed to butachlor for 30 days [34]. The maximum concentration of $1.80 \mathrm{E}-01 \mathrm{mg} / \mathrm{kg}$ detected in the fish sample from the present study was lower than the findings of Yadav et al. [35], which revealed 1.0 $\mathrm{mg} / \mathrm{kg}$ butachlor and suggested that butachlor interfered with cellular activities in fishes at genetic level, inducing chromosomal aberrations suggesting a serious concern towards the potential danger of butachlor for aquatic organisms. Hence, the studied herbicide residues in the fish samples were significantly higher in the liver and gills of all the fish studied than other organs. Such high levels are due to the fact that fresh water fishes' gills might be expected to be the primary route for the uptake of water pollutants; while the liver serves as a storage organ for a vast variety of nutrients. High accumulation of the herbicides in the gills and liver may also be as a result of detoxicating mechanisms and may originate from pesticides deposited in the sediments and food in the aquatic environment. However, the liver is the preferred organ for herbicide accumulation as could be deduced from the present study. Accumulation of pesticides in different species of fish is the function of their respective membrane permeability and enzyme system, which is highly species specific and also the herbicide residues accumulated in different organs in the fish as observed in the study.

\section{Conclusion}

The concentrations of all the herbicides in the studied samples water, sediment and tissues of fish were observed to be higher during the rainy season. Atrazine was the most predominant herbicide in all the samples studied. Accumulation of herbicides in the studied fish species were in the order of Synodontis budgetti>Heterotis niloticus $>$ Tilapia zilli $>$ Clarius anguillarias. Accumulation of herbicides in the tissues of the fish samples studied were in the order of liver $>$ gills $>$ intestine $>$ flesh. All the studied herbicides were found to be within the ADI value of 0.05 $\mathrm{mg} / \mathrm{kg}$ in fish and $0.01 \mathrm{mg} / \mathrm{kg}$ MRLs in fish samples.

\section{References}

1. United State Environmental Protection Agency-USEPA (2006) Decision documents for atrazine.

2. Federal Environmental Protection Agency-FEPA (2003) Guidelines and standards for environmental pollution control in nigeria.

3. Haney RL, Senseman SA, Hons FM, Zuberer DA (2000) Effect of glyphosate on soil microbial activity and biomass. Weed Sci 48: 89-93.

4. Derksen DA, Anderson RL, Blackshaw RE, Maxwell B (2002) Weed dynamics and management strategies for cropping systems in the Northern Great Plains. Agron J 94: 174-185.

5. Faheed FA, Abd-Elfattah Z (2007) Alteration in growth and physiologica activities in Chlorella vulgaris under the effect of photosynthetic inhibitor diuron. Int J Agric Biol 9: 631-634.

6. Directorate of Fertilizer and Pesticide- DFP (2011) Pesticides for Agriculture and Forestry.

7. Wahyu W, Rosli BM, Adam BP, Dzolkhifli O, Abdul SJ, et al. (2009) Residua phytotoxicity effects of paraquat, glyphosate and glufosinate-ammonium herbicides in soil from field-treated plots. Int J Agric Biol 11: 214-216.

8. Ouyang Y, Mansell RS, Nkedi-Kizza P (2004) A simple high-performance liquid chromatography method for analyzing paraquat in soil solution samples. J Environ Qual 33: 406-408.
9. Vörösmarty CJ, Sahagian D (2000) Anthropogenic disturbance of the terrestrial water cycle. Biosci 50: 753-765

10. Syvitski JPM, Vörösmarty CJ (2005) Impact of humans on the flux of terrestrial sediment to the global coastal ocean. Science 308: 376-380.

11. Mackenzie FT, Vera LM (2002) Century-scale nitrogen and phosphorus controls of the carbon cycle. Chem Geol 190: 13-32.

12. Canfield DE, Glazer AN (2010) The evolution and future of earth's nitrogen cycle. Science 330: 192-196.

13. Fabricius KE (2011) Factors determining the resilience of coral reefs to eutrophication: A review and conceptual model, Coral Reefs: An Ecosystem in Transition, Springer, Berlin, Germany.

14. Coles S, Jokiel P (1992) Effects of salinity on coral reefs, Pollution in tropical aquatic systems. CRC Press Incorporation, Florida, USA.

15. De'ath, G, Fabricius K, Sweatman H, Puotinen M (2012) The 27year decline of coral cover on the Great Barrier Reef and its causes. Proceedings of the National Academy of Sciences, USA.

16. Fabricius KE, Okaji K, Death G (2010) Three lines of evidence to link outbreaks of the crown-of-thorns seastar Acanthaster planci to the release of larval food limitation. Coral Reefs 29: 593-605.

17. Hughes TP, Graham NAJ (2010) Rising to the challenge of sustaining coral reef resilience. Trends Ecol Evolut 25: 633-642.

18. Mumby PJ, Steneck RS (2011) The resilience of coral reefs and its implications for reef management, New Coral Reefs: An Ecosystem in Transition, Springer, Berlin, Germany.

19. Wooldridge SA, Done TJ (2009) Improved water quality can ameliorate effects of climate change on corals. Ecol Appl 19: 1492-1499.

20. Consensus Statement on Climate Change and Coral Reefs-CSCCCR (2012) The $97 \%$ consensus on global warming

21. Boyd CE, Tucker CS (1992) Water quality and pond soil analyses for aquaculture. Alabama Agricultural Experiment Station, Auburn University, USA

22. Paula P, Michelangelo A, Dorothea M, Irina S, Bünyamin T, et al. (2007) Analysis of pesticide residues using the Quick Easy Cheap Effective Rugged and Safe (QuEChERS) pesticide multiresidue method in combination with gas and liquid chromatography and tandem mass spectrometric detection. Anal Bioanal Chem 389: 1697-714.

23. Kim KS, Lee SC, Kim KH, Shim WJ, Hong SH (2009) Survey on organ chlorine pesticides, PCDD/Fs, dioxin-like PCBs and HCB in sediments from the Han River Korea. Chemosphere 75: 580-587.

24. Eisler R (1990) Paraquat hazards to fish, wildlife, and invertebrates: A synoptic review, U.S. Fish and Wildlife Service Patuxent Wildlife Research Center Laurel, Maryland,USA.

25. Fernandez M, Ibanez M, Pico Y, Manes J (1998) Spatial and temporal trends of paraquat, diquat and difenzoquat contamination in water from marsh areas of the Valencian Community (Spain). Arch Environ Contam Toxicol 35: 377-384.

26. Knickmeyer $R$, landgraff $O$, Steinhart H (1992) Cyclic Organochlorines in the sea star asterias rubens from the German bight, December 1988-May 1989. Marine Environmental Research 33: 127-143.

27. Ezemonye IT (2009) Lethal and sublethal effects of atrazine to amphibian larvae Jordan. Jordan J Biol Sci 1: 29-36.

28. Hopenhayn-rich ML, Stump SR (2001) Regional assessment of atrazine exposure and incidence of breast and ovarian cancers in Kentucky. Arch Environ Contam Toxicol 42: 127-136

29. Peighambarzadeh Z, Safi S, Shahtaheri SJ, Javanbakht M, Forushani AR (2011) Presence of atrazine in the biological samples of cattle and its consequence adversity in human health. Iran J Public Health 4: 12-121.

30. He H, Chen G, Yu J, He J, Huang X, et al. (2013) Individual and joint toxicity of three chloroacetanilide herbicides to freshwater Cladoceran Daphnia carinata. Bull Environ Contam Toxicol 90: 344-350.

31. Zheng J, Li R, Zhu J, Zhang J, He J, et al. (2012) Degradation of the chloroacetamide herbicide butachlor by Catellibacteriumcaeni nov DCA-1T. Int Biodeterior 73: 16-22.

32. Muley DV, Karanjkar DM, Maske SV (2007) Impact of industrial effluents on the biochemical composition of freshwater fish Labeo-rohita. J Environ Biol 28: 245249 . 
Citation: Mohammed AI, Akan JC, Chellube Z, Inuwa LB (2018) Fate of Komadugu River Basin, Yobe State, Nigeria With Respect to Herbicides Residues. J Pet Environ Biotechnol 9: 383. doi: 10.4172/2157-7463.1000383

Page 7 of 7

33. Rajput V, Singh S K, Kirti A, Abhishek P (2012) Comparative toxicity of Butachlor, Imidacloprid and Sodium fluoride on protein profile of the walking cat fish Clarias batrachus. J Appl Pharm Sci 2: 121-124.

34. Chang J, Liu S, Zhou S, Wang M, Zhu G (2013) Effects of butachlor on reproduction and hormone levels in adult zebrafish (Daniorerio). Exp Toxicol Pathol 65: 205-209.

35. Yadav A, Bhatnagar A, Kaur M (2013) Aberrations in the chromosomes of Cirrhinus mrigala (Hamilton) upon exposure to butachlor. J Toxicol 7: 858-865. 